\title{
Características Físicas da Digesta e Resistência de Partículas ao Escape do Rúmen- Retículo em Novilhos Mantidos em Pastagem Natural em Diferentes Estações do Ano ${ }^{1}$
}

\author{
José Carlos Pereira ${ }^{2}$, Marcelo Suzart de Almeida ${ }^{3}$, Augusto César de Queiroz ${ }^{2}$, \\ Paulo Roberto Cecon ${ }^{4}$, Sérgio Pereira Braz ${ }^{5}$
}

\begin{abstract}
RESUMO - O tamanho médio de partículas (TMP) e módulo de finura (MF) da dieta, da digesta ruminal e das fezes de novilhos, além da resistência relativa $(\mathrm{RR})$ das partículas ao escape do rúmen-retículo, foram estudados em uma pastagem natural da Zona da Mata de Minas Gerais, durante a Época 1 (E1), que abrangeu os meses fevereiro/março/abril, e Época 2 (E2), correspondente aos meses de agosto/setembro/outubro. O TMP e MF da dieta selecionada pelos bovinos não variaram entre a E1 $(1,91 \mathrm{~mm}$ e 3,62) e E2 (2,10 mm e 3,72). Na E1, o TMP e MF da digesta ruminal decresceram linearmente até o tempo de 24 horas, enquanto na E2 apresentaram comportamento quadrático, aumentando até o máximo de 1,62 mm e 3,31 nos tempos de 13,20 e 12,95 horas, respectivamente, após início da alimentação. Para o TMP e MF das fezes, não houve efeito do tempo de coleta, mas foram diferentes entre E1 (0,55 mm e 2,09) e E2 $(0,45 \mathrm{~mm}$ e 1,90$)$ e nos meses dentro da E1. A redução do tamanho das partículas na E2 foi um fator limitante regulando a saída da digesta do rúmen, sendo que na E1 favoreceu o escape de partículas de tamanho médio. Ocorreu aumento contínuo na resistência ao escape das partículas do rúmen com o aumento do tamanho das partículas; partículas maiores que 1,19 mm escaparam do rúmen, apesar de enfrentarem resistência, e as partículas menores que este tamanho sofreram diferentes graus de resistência ao escape.
\end{abstract}

Palavras-chave: módulo de finura, pastagem natural, resistência relativa, tamanho de partícula

\section{Physical Characteristics of Digesta and Resistance of Particles to Escape of Rumen- Reticulum in Steers Grazing Native Pasture, in Different Seasons of the Year}

\begin{abstract}
The average particle size (PS) and module of fineness (MF) of the diet, ruminal digesta and feces of steers, as well as relative resistance (RR) of the particles to escape of the reticulum-rumen, were studied in a native pasture of the Zona da Mata - Minas Gerais State, during the Season 1 (S1), that corresponded to the months of February/March/April, and Season 2 (S2), that corresponded to the months of August/September/October. The PS and MF of the diet selected by the bovine did not vary among the $\mathrm{S} 1$ (1.91 $\mathrm{mm}$ and 3.62) and the S2 showed (2.10 mm and 3.72). In the S1, PS and MF of the ruminal digesta linearly decreased until the 24 hours time, while, in the S2, presented a quadratic response, increasing up to a maximum of $1.62 \mathrm{~mm}$ and $3.31 \mathrm{in}$ the 13.20 and 12.95 hours, respectively, after beginning of the feeding. For PS and MF of the feces, there was no effect of the collection time, but it were different between the E1 $(0.55 \mathrm{~mm}$ and 2.09) and E2 $(0.45 \mathrm{~mm}$ and 1.90$)$ and in the months within the E1 season. The reduction of the particles sizes in the $\mathrm{E} 2$ was a restriction factor regulating the exit of digesta from the rumen, and in the E1, it favored the escape of medium particle sizes. A continuous increase in the resistance of the particles to escape from the rumen with the increase of the particle size was noticed; particles larger than $1.19 \mathrm{~mm}$ escaped from the rumen, in spite of face strong resistance, and the smaller particles than this size faces different degrees of resistance to escape from the rumen.
\end{abstract}

Key Words: module of fineness, native pasture, particle size, relative resistance

\section{Introdução}

Para alcançar digestão efetiva da fração fibrosa da dieta, os ruminantes, durante seu processo evolutivo, desenvolveram a capacidade para reter partículas do alimento, potencialmente digestíveis e indigestíveis, para sofrerem o processo de redução de tamanho e digestão, tornando-as aptas a passar pelos compartimentos do estômago. Assim, a redução do tamanho das partículas, pela mastigação inicial, libera os nutrientes solúveis para fermentação, mediante remoção da cutícula, separação dos tecidos da forragem e exposição da estrutura interna da forragem para invasão efetiva dos microrganismos do rúmen-retículo (Ulyatt et al., 1986).

\footnotetext{
${ }_{1}^{1}$ Parte da tese de Doutorado apresentada pelo segundo autor à UFV, parcialmente financiada pelo CNPq.

${ }^{2}$ Professor Titular do Departamento de Zootecnia da UFV, Bolsista do CNPq. E.mail: jcarlos@mail.ufv.br

3 Professor da UNIPAR, Umuarama-PR. E.mail: suzart@unipar.com.br

${ }^{4}$ Professor Adjunto do Departamento de Informática da UFV.

${ }^{5}$ Estudante de Doutorado da UFRRJ.
} 
Segundo Poppi et al. (1980), a produtividade dos ruminantes não depende somente da qualidade da dieta e do consumo voluntário, mas também da taxa de redução do tamanho de partícula da digesta ou da facilidade de mastigação durante a ruminação, que é uma propriedade da composição da dieta. Esta dieta inclui principalmente o conteúdo da parede celular e a propriedade física da fibra, que influenciam na quebra em partículas de fibra menores (Van Soest, 1994). Assim, nos estudos da cinética de degradação do alimento nos ruminantes, deve-se conhecer não somente a anatomia e fisiologia do trato gastrintestinal (TGI), mas também as propriedades físicas do alimento e da digesta (Udén \& Van Soest, 1982).

A mastigação durante a ruminação promove a exposição da estrutura interna da planta para o ataque microbiano e reduz o tamanho das partículas (Dulphy et al., 1980), sendo de grande importância para a redução contínua do tamanho das partículas grandes (Poppi et al., 1981; Lee \& Pearce, 1984; Ulyatt et al., 1986; Van Soest et al., 1988; e McLeod \& Minson, 1988 a,b). Portanto, essa ruptura das partículas do alimento até um tamanho limite que lhes permita atravessar o orifício retículo-omasal, estabelecido em 1,18 mm no caso de ovelhas (Poppi et al., 1980), e 1,5-2,0 mm para bovinos (Ulyatt et al., 1986), está em função de quatro mecanismos: a mastigação durante o processo de preensão dos alimentos; a mastigação posterior, durante a ruminação; a digestão microbiana; e a ruptura devido à fricção provocada pelas paredes do rúmen, sendo os dois primeiros os principais responsáveis (Faichney, 1986).

A atividade microbiana pouco contribui para degradação física das partículas fibrosas (Van Soest, 1982; Kennedy, 1985; Ulyattet al., 1986). Entretanto, indiretamente, a fermentação microbiana tem contribuição substancial na redução do tamanho da partícula pelo aumento na fragilidade das partículas fibrosas, aumentando, assim, a eficiência da quebra durante a ruminação (Chai et al., 1984; McLeod \& Minson, 1988a).

Faichney (1986) descreve o modelo australiano em que se postula tamanho limite das partículas que abandonam o retículo-rúmen entre 1-2 mm, enquanto o modelo americano postula um tamanho de 3-4 mm. Este modelo, desenvolvido por Mertens et al. (1984), citado por Van Soest et al. (1988), tenta avaliar o efeito da redução do tamanho das partículas no fluxo da digesta e apresenta três pools de partículas: grandes, médias e pequenas, as quais contêm frações disponíveis e não-disponíveis, sendo a disponibilidade definida como o material da dieta potencialmente digestível. A diferença no tamanho limite entre os modelos pode estar relacionada ao fato de o modelo americano ter sido desenvolvido para vacas de leite de alta produção, sem considerar diferenças de consumo com animais não-lactantes, e o modelo australiano estar centrado em condições de alimentação e características morfológicas das forrageiras que podem influenciar a digesta.

A idéia do conceito de tamanho crítico de partícula (Balch \& Campling, 1962 e Greenhalgh \& Reid, 1973) advém do tamanho limite das partículas para escapar do retículo-rúmen, dividindo as partículas da digesta em dois grupos distintos: um de partículas grandes, que enfrentam alta resistência relativa em abandonar o retículo-rúmen, e o outro de partículas pequenas, que fluem com relativa facilidade (Baldwin et al., 1977; Poppi et al., 1980).

O fato de as partículas poderem ou não passar para o omaso depende principalmente da probabilidade de estarem presentes na região ventral do retículo durante a abertura do orifício retículo-omasal (Reid, 1984). A maior concentração de partículas pequenas e de maior densidade, presentes na região ventral do retículo, em relação ao rúmen ventral e dorsal, é a razão para o fluxo preferencial deste tipo de partícula (Sutherland, 1988).

Vários processos contribuem no decréscimo da possibilidade de escape das partículas grandes, sendo o embaraço na malha fibrosa no rúmen, o efeito de filtragem na passagem do rúmen para o retículo, o efeito de peneiragem dentro da massa reticular ou no orifício retículo-omasal e o efeito de filtragem dentro do omaso, devido ao refluxo, fatores em potencial que contribuem para a diminuição da probabilidade de escape das partículas grandes do retículo-rúmen (Sutherland, 1988).

Diversas técnicas têm sido estudadas para subdividir as partículas de amostras do alimento e da digesta em frações de tamanho definido. A técnica do peneiramento seco, com base na utilizada para solos e fertilizantes, foi padronizada para alimentos moídos e triturados (ASAE, 1969a,b; Ensor et al., 1970). Smith \& Waldo (1969) modificaram o procedimento da FDN de Van Soest para fazer uma preparação não destrutiva das partículas de amostra da digesta seca, removendo a interferência do efeito cimentante da proteína, separando os agregados sem uso de força mecânica, eliminando a possível degradação do ta- 
manho da partícula e aplicando posteriormente a técnica do peneiramento seco.A técnica do peneiramento úmido foi desenvolvida para o fracionamento de amostra da digesta (Evans et al., 1973; Poppi et al., 1980) e o alimento deglutido e coletado no esôfago (Reid et al., 1977). A principal vantagem desta técnica é que a distribuição das partículas da digesta é determinada no estado normal de hidratação, sendo a quebra de partícula durante o peneiramento menos provável (Faichney, 1986). Todavia, as partículas de forma alongada escorrem entre os poros da peneiras e são retidas em peneiras cujo tamanho do poro não tem relação com o tamanho da partícula. McLeod et al. (1990) observaram que peneira com poro de $1,18 \mathrm{~mm}$, usada para separar partículas grandes de pequenas, retém partículas medindo acima de $10 \mathrm{~mm}$ de comprimento, mas permitem a passagem de partículas de $5 \mathrm{~mm}$.

Devido à existência de poucas informações, na literatura, sobre as características físicas da digesta de novilhos em pastejo, o presente trabalho foi conduzido para avaliar o tamanho médio de partícula e o módulo de finura da dieta, da digesta e das fezes de novilhos, bem como a resistência relativa das partículas ao escape do rúmen-retículo, em um pasto natural da Zona da Mata de Minas Gerais.

\section{Material e Métodos}

O experimento foi conduzido, em uma área de pastagem natural, localizada na Zona da Mata de Minas Gerais, constituída por diversas espécies de gramíneas, tendo como predominantes o capim-gordura (Melinis minutiflora Pal. de Beauv), capimsapé (Imperata brasiliensis Trin.), capim-jaraguá (Hyparrhenia rufa (Nees) Stapf) e grama-batatais (Paspalum notatum Flugge); e leguminosas como o desmodium (Desmodium spp.), stylosantes (Stylosanthes sp.), puerária (Pueraria phaseoloides (Roxb.) Benth) e soja-perene (Neonotonia wightii L.), além de ervas e arbustos típicos da região.

O período experimental foi dividido em duas épocas com três meses cada: Épocal (E1), representando um período em que ocorria declínio gradativo da temperatura e da precipitação, compreendendo os meses de fevereiro, março e abril, e Época 2 (E2), representando uma situação inversa da anterior, abrangendo os meses de agosto, setembro e outubro. Foram utilizados cinco bovinos mestiços HolandêsZebú, de aproximadamente 30 meses de idade, pe- sando entre 350 e $400 \mathrm{~kg}$, fistulados no esôfago e no rúmen. Os animais ficaram livres para o pastejo, durante todo o período experimental, excetuando os momentos de coleta de amostras, e durante o jejum prévio para a coleta da extrusa, e tinham acesso a mistura mineral e água, não sendo utilizada alimentação adicional.

As amostras da extrusa foram coletadas em bolsas de náilon, contendo um fundo de tela plástica (malha rígida), para drenagem do excesso de saliva, após jejum prévio dos animais de aproximadamente 16 horas, e com duração máxima de 30 minutos/dia.

Devido às dificuldades metodológicas para o esvaziamento do rúmen, amostras de digesta ruminal, modificando o procedimento de Pearce (1967), sucederam-se após jejum, nos tempos de $4 ; 8 ; 12$ e 24 horas, a partir da primeira alimentação. Para se obter amostras mais representativas da digesta ruminal, sub-amostras foram retiradas do retículo, e dos sacos cranial, dorsal e ventral do rúmen. As amostras de fezes seguiram os mesmos tempos anteriores, após decorrido um tempo de 24 horas da coleta de digesta.

Para determinação da granulometria, as amostras foram processadas segundo a metodologia descrita por Smith \& Waldo (1969), com algumas adaptações, e uma vez processadas, procedeu-se ao peneiramento em equipamento PRODUTEST com rotor na posição 10 e tempo de peneiramento de 10 minutos, suficiente para alcançar estabilidade de conteúdo em cada peneira, de acordo com os procedimentos adotados pela ADSA (1970). As peneiras utilizadas tinham aberturas de 0,$149 ; 0,297 ; 0,590$; 1,190; 2,380; e 4,760 mm, $20 \mathrm{~cm}$ de diâmetro e $5 \mathrm{~cm}$ de profundidade. $O$ peso seco retido em cada peneira foi expresso em porcentagem do peso seco total retido nas seis peneiras, enquanto o peso seco acumulado foi calculado para cada peneira como a proporção de peso seco total que seria retida por esta peneira, ou seja, o peso seco retido em uma peneira em particular, mais aquele retido nas peneiras de tamanho do poro imediatamente superiores, expresso também em porcentagem.

Para caracterização de tamanho da partícula da dieta, da digesta ruminal e das fezes, foi utilizado o tamanho médio de partícula (TMP), calculado por intermédio de parâmetros da equação exponencial sugerida por Pond et al. (1984), citados por Fischer et al. (1988):

$$
\mathrm{R}=100 * \mathrm{e}^{(-\mathrm{K} *(\mathrm{~S}-\mathrm{W}))}
$$

em que: R é a porcentagem acumulada do peso seco da amostra por peneira; S é o tamanho da abertura do 
poro da peneira $(\mathrm{mm})$; W é o menor tamanho de partícula estimado ( $\mathrm{mm}$ ); e K é o declínio constante da curva exponencial, que indica a proporção de partículas acima de algum tamanho de tela que passará uma peneira mais larga.

Assim, o TMP pode ser calculado da seguinte forma: $\mathrm{TMP}=1 / \mathrm{K}+\mathrm{W}$

Para caracterizar o tamanho da partícula, utilizou-se também o módulo de finura (MF), determinado de acordo com a metodologia recomendada pela ASAE (1969a,b) e adotada pela ASAS (1969).

O módulo de finura é uma medida adimensional que expressa a finura da amostra, calculada pelo somatório do produto, em porcentagem, entre a porcentagem de peso seco retido sobre cada peneira e um coeficiente. Este variou em ordem decrescente de 6 a 1, da maior para a menor peneira.

A resistência relativa (RR) ao escape das partículas, para fora do rúmen, foi calculada assumindose que as partículas retidas na peneira de abertura de poro igual a $0,149 \mathrm{~mm}$ passam sem resistência, conforme a fórmula descrita por Poppi et al. (1980):

$$
R R(\%)=100\left(1-\frac{B r^{*} Y f}{B f^{*} Y r}\right)
$$

em que: RR (\%) é a resistência relativa das partículas ao escape do rúmen, para cada peneira usada; $\mathrm{Br}$ e $\mathrm{Bf}$ é a porcentagem das partículas, do rúmen e das fezes, retidas na peneira de referência $(0,149$ $\mathrm{mm})$ no rúmen e fezes; e Yr e Yf é a porcentagem de partículas, do rúmen e das fezes, retidas na peneira, cuja resistência está sendo calculada.

Para o TMP e MF da dieta (extrusa) avaliados, foi feita análise de variância, em delineamento experimental inteiramente casualizado, com cinco repetições e meses dentro de cada época.

Para aplicação dos dados à equação exponencial, foi utilizado o algoritmo Quasi-Newton, do procedimento Nonlinear Estimation do programa STATISTICA versão 5.0.

Para TMP e MF da digesta ruminal e das fezes e resistência relativa das partículas ao escape do retículo-rúmen, foi efetuada análise de variância em delineamento inteiramente casualizado, com quatro tempos de amostragem, cinco repetições e três meses dentro de cada época.

Para o processamento da análise de variância dos dados, foi utilizado o programa SAEG, versão 7.0, enquanto para comparação de médias foi utilizado o teste Newman-keuls (SNK) a 5\% de probabilidade.

\section{Resultados e Discussão}

O tamanho médio de partícula (TMP) e o módulo de finura (MF) da dieta selecionada pelos bovinos não variaram entre a E1 $(1,91 \mathrm{~mm}$ e 3,62$)$ e a E2 $(2,10 \mathrm{~mm}$ e 3,72 ), como também entre os meses dentro de cada época.

Martz \& Belyea (1986) sugeriram que a forragem é mastigada a tamanhos de 1 a $2 \mathrm{~mm}$, antes de ser deglutida, enquanto Fisher et al. (1988), trabalhando com gramíneas tropicais, encontraram valores de 1,96 $\mathrm{mm}$ para TMP.

Neste estudo, o peso seco acumulado nas peneira apresentou $26 \%$ de partículas mastigadas grandes $(\geq 2,38 \mathrm{~mm}), 56 \%$ médias $(<2,38$ e $\geq 0,59 \mathrm{~mm})$ e $18 \%$ pequenas $(<0,59 \mathrm{~mm})$, semelhantes às encontradas por Burns et al. (1992), que também trabalharam com gramínea tropical durante a estação de crescimento.

A mastigação durante a ingestão é um processo bastante eficiente para redução do tamanho de partícula em forragens verdes, considerando que $50 \%$ da MS do alimento pode ser reduzida a um tamanho inferior a $1 \mathrm{~mm}$ em apenas um período de mastigação (Ulyatt, 1983), embora haja variações entre dietas.

Ulyatt et al. (1986) verificaram que dietas verdes ou frescas e aquelas com valor nutritivo alto eram mastigadas de forma mais eficiente que as dietas secas e com baixo valor nutritivo.

Houve efeito $(\mathrm{P}<0,05)$ da interação época ou estação e tempo de coleta da digesta no rúmen na estimação do tamanho médio de partícula (TMP) e do módulo de finura (MF), como pode ser visto na Tabela 1.

Observa-se diferença $(\mathrm{P}<0,05)$ nos dois parâmetros citados, entre as épocas, para os tempos de 4 e 8 horas, e nenhuma diferença para os tempos de 12 e 24 horas. Os valores do TMP e MF foram menores na $\mathrm{E} 2(1,19 \mathrm{~mm}$ e 2,98 e 1,35 $\mathrm{mm}$ e 3,15) em relação a $E 1(1,77 \mathrm{~mm}$ e 3,44 e 1,69 $\mathrm{mm}$ e 3,35) respectivamente para os tempos de 4 e 8 horas; sendo que a partir das 12 horas após início da alimentação essa proporção se equilibra e, conseqüentemente, os valores de TMP e MF se igualam entre as épocas.

$O$ efeito da taxa de redução do tamanho das partículas, após a alimentação, entre as épocas, pode ser observado pelas equações linear e quadrática do tamanho médio das partículas e do módulo de finura, em função do tempo de coleta, apresentadas na Figura 1. Nota-se que durante o verão o TMP e MF 
Tabela 1 - Tamanho médio de partícula (TMP) e módulo de finura (MF) da digesta ruminal de novilhos em função dos tempos de amostragem da digesta

Table 1 - Mean particle size (MPS) and module of fineness (MF) of the ruminal digesta of the steers, in funtion of sampling times of the digesta

\begin{tabular}{|c|c|c|c|c|c|c|c|c|}
\hline \multirow[t]{4}{*}{$\begin{array}{l}\text { Época } \\
\text { Season }\end{array}$} & \multicolumn{8}{|c|}{$\begin{array}{l}\text { Tempos de amostragem-horas } \\
\text { Sampling times - hours }\end{array}$} \\
\hline & \multicolumn{2}{|c|}{4} & \multicolumn{2}{|c|}{8} & \multicolumn{2}{|c|}{12} & \multicolumn{2}{|c|}{24} \\
\hline & TMP & $\mathrm{MF}$ & TMP & $\mathrm{MF}$ & TMP & $\mathrm{MF}$ & TMP & $\mathrm{MF}$ \\
\hline & $M P S$ & $F M$ & $M P S$ & $F M$ & $M P S$ & $F M$ & $M P S$ & $F M$ \\
\hline $\mathrm{E} 1(S 1)$ & $1,77^{\mathrm{a}}$ & $3,44^{\mathrm{a}}$ & $1,69^{\mathrm{a}}$ & $3,35^{\mathrm{a}}$ & $1,54^{\mathrm{a}}$ & $3,31^{\mathrm{a}}$ & $0,95^{\mathrm{a}}$ & $2,75^{\mathrm{a}}$ \\
\hline $\mathrm{E} 2(\mathrm{~S} 2)$ & $1,19^{b}$ & $2,98^{\mathrm{b}}$ & $1,35^{\mathrm{b}}$ & $3,15^{b}$ & $1,70^{\mathrm{a}}$ & $3,34^{\mathrm{a}}$ & $0,97^{\mathrm{a}}$ & $2,76^{\mathrm{a}}$ \\
\hline
\end{tabular}

Médias seguidas por letra diferente, na coluna, diferem $(P<0,05)$ pelo teste SNK.

Means followed by the different letter in the column differ $(P<.05)$ by SNK test.

da digesta no rúmen decrescem linearmente até o tempo de 24 horas e, durante o inverno, apresentaram comportamento quadrático, aumentando até um máximo de 1,62 mm e 3,31 nos tempos de 13,20 e 12,95 horas, respectivamente, após início da alimentação. Portanto, nas condições do presente estudo, durante a E1, os animais conseguiram consumir mais forragem, nas primeira horas após início da alimentação, e iniciaram um processo efetivo de redução do tamanho das partículas, a partir desse momento. Já na E2, provavelmente, os animais não conseguem saciar a

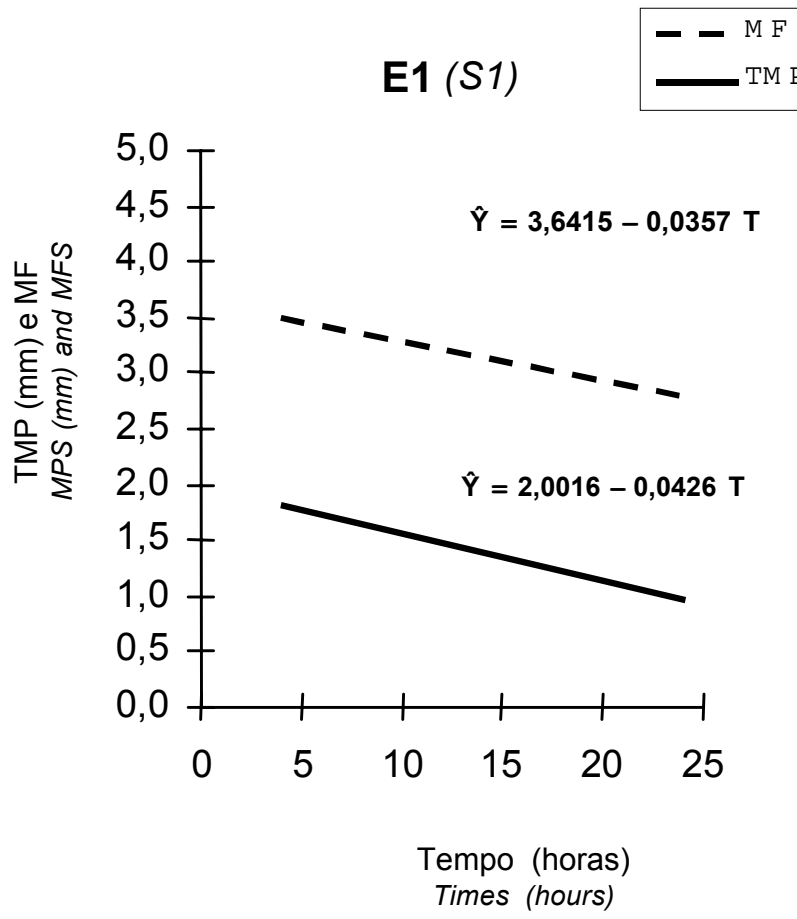

capacidade do rúmen nas primeiras horas, tendo efetiva redução do tamanho das partículas no rúmen somente 13 horas após o início da alimentação. Isso vem confirmar ainda mais a hipótese da habilidade seletiva dos bovinos, quando submetidos a pastejo, gastando mais tempo a procura de uma dieta de melhor qualidade (Hodgson, 1982; Cosgrove, 1997).

Waghorn et al. (1989) observaram ritmo de redução mais acentuado em leguminosas que em gramíneas, principalmente nas três primeiras horas após o fornecimento das dietas.

Figura 1 - Estimativa do tamanho médio de partícula (TMP) e do módulo de finura (MF) da digesta do rúmen de novilhos, em função do tempo ( $T$ ) de amostragem.

Figure 1 - Estimate of mean particle size (MPS) and module of fineness (MF) of the ruminal digesta of the steers, in funtion of sampling times of the digesta.

R. Bras. Zootec., v.31, n.2, p.731-739, 2002 
Luginbuhl et al. (1990), em trabalho com feno de capim-coastcross (Cynodon dactylon), relataram que, ao aumentar o tempo após alimentação, houve decréscimo linear na porcentagem de partículas grandes, com aumento linear e quadrático nas porcentagens de partículas pequenas e finas, respectivamente, da digesta do extrato superior do rúmen.

$\mathrm{Na}$ Tabela 2, observa-se diferença $(\mathrm{P}<0,05)$ nos meses dentro da E1, para os TMP e MF da digesta no rúmen, e entre estações, ficando a média da $\mathrm{E} 1 \mathrm{em}$ $1,49 \mathrm{~mm}$ e 3,21 e a da E2 em 1,30 $\mathrm{mm}$ e 3,06, respectivamente. A menor fração indegradável da FDN da dieta dos novilhos no pasto natural, na E1, possivelmente facilita a redução do tamanho das partículas ingeridas, de forma linear (Figura 1), favorecendo maior consumo voluntário e oportunidade de escape às partículas médias (Figura 2). Portanto, a mastigação durante a ruminação sofre efeito do conteúdo da parede celular, como destaca Van Soest (1994), pois, em outro estudo nesta pastagem, foi encontrado elevado teor de FDN na dieta selecionada na E1 e E2, sendo que na E2 a fração indegradável da FDN na dieta selecionada foi superior.

Para o TMP e MF das fezes, não houve efeito do tempo de coleta, mas foram diferentes $(\mathrm{P}<0,01)$ entre a E1 $(0,55 \mathrm{~mm}$ e 2,09$)$ e a E2 $(0,45 \mathrm{~mm}$ e 1,90$)$ e nos meses dentro da E1 (Tabela 3). As partículas médias $(<2,38$ e $\geq 0,59 \mathrm{~mm})$ representam $56 \%$ das partículas ingeridas, na E1 e E2; e a menor resistên-

Tabela 2 - Tamanho médio da partícula (TMP) e módulo de finura (MF) da digesta do rúmen de novilhos

Table 2 - Mean particle size (MPS) and module of fineness (MF) of the ruminal digesta of the steers

\begin{tabular}{|c|c|c|c|c|c|}
\hline & \multicolumn{2}{|c|}{$\begin{array}{l}\text { Época } 1 \\
\text { Season } 1 \\
\end{array}$} & & \multicolumn{2}{|c|}{$\begin{array}{l}\text { Época } 2 \\
\text { Season } 2 \\
\end{array}$} \\
\hline & $\begin{array}{l}\text { TMP } \\
M P S\end{array}$ & $\begin{array}{l}\text { MF } \\
F M\end{array}$ & & $\begin{array}{l}\text { TMP } \\
M P S\end{array}$ & $\begin{array}{l}\text { MF } \\
F M\end{array}$ \\
\hline $\begin{array}{l}\text { Fevereiro } \\
\text { February }\end{array}$ & $1,41^{\mathrm{b}}$ & $3,16^{\mathrm{b}}$ & $\begin{array}{l}\text { Agosto } \\
\text { August }\end{array}$ & $1,24^{\mathrm{a}}$ & $3,02^{\mathrm{a}}$ \\
\hline $\begin{array}{l}\text { Março } \\
\text { March }\end{array}$ & $1,30^{\mathrm{b}}$ & $3,08^{b}$ & $\begin{array}{l}\text { Setembro } \\
\text { September }\end{array}$ & $1,19^{\mathrm{a}}$ & $3,00^{\mathrm{a}}$ \\
\hline $\begin{array}{l}\text { Abril } \\
\text { April }\end{array}$ & $1,76^{\mathrm{a}}$ & $3,40^{\mathrm{a}}$ & $\begin{array}{l}\text { Outubro } \\
\text { October }\end{array}$ & $1,48^{\mathrm{a}}$ & $3,17^{\mathrm{a}}$ \\
\hline $\begin{array}{l}\text { Média } \\
\text { Mean }\end{array}$ & $1,49^{\mathrm{A}}$ & $3,21^{\mathrm{A}}$ & $\begin{array}{l}\text { Média } \\
\text { Mean }\end{array}$ & $1,30^{\mathrm{B}}$ & $3,06^{\mathrm{B}}$ \\
\hline
\end{tabular}

Médias seguidas por letra minúscula diferente, na coluna, diferem $(P<0,05)$ pelo teste SNK (Meansfollowed by different small letterin the column differ $[P<.05]$ by SNK test).

Médias seguidas por letra maiúscula diferente, na linha, diferem $(P<0,01)$ pelo teste $F$ (Means followed by different capital letters in the row differ $[P<.01]$ by $S N K$ test). cia ao escape do rúmen na E1 (Figura 2) proporciona maior tamanho das partícula nas fezes. Estes resultados mostram que maior consumo voluntário de pasto e maior taxa de passagem durante a E1, observados em outro estudo nesta pastagem, de certa forma estão relacionados a redução linear do tamanho das partículas após início da alimentação (Figura 1).

Nesse sentido, a ocorrência de maior fração indegradável no pasto e o exercício da habilidade seletiva dos bovinos, à procura de um alimento de melhor qualidade, induzem a redução do consumo voluntário no inverno(Hodgson, 1982; Hoyos \& Lascano, 1988; Pereira et al., 1992; Minson \& Wilson, 1994; Cosgrove, 1997).

Poppi et al. (1980) encontraram, para as forrageiras Lotononis bainesii, Stylosanthes guyanensis, Digitaria decumbens, Paspalum plicatulum e Brachiaria decumbens, valores para o módulo de finura da digesta de ovinos no rúmen variando de 2,29 a 2,83 e nas fezes de 1,61 a 1,98. Esses valores, menores que os deste estudo, resultam da maior capacidade de mastigação dos ovinos em relação aos bovinos (Van Soest, 1994).

A teoria do tamanho crítico para as partículas escaparem do rúmen-retículo, assume que partículas acima de certo tamanho não podem escapar do rúmen, o que significa que todas partículas menores que o tamanho crítico saem com a mesma facilidade.

O gráfico do peso seco acumulado, apresentado na Figura 2, mostra que partículas retidas pela peneira de $1,19 \mathrm{~mm}$ podem sair do rúmen, contribuindo, em média, com 9,8\% das partículas que escapam. Esta observação não seria esperada se forem considerados os trabalhos de Pearce (1967), Smith et al. (1967) e Reid et al. (1977), mas, levando em conta o maior conteúdo de fibra das forrageiras tropicais, pode ocorrer maior oportunidade para as partículas grandes mostrarem sua habilidade de escapar do rúmen.

Foi considerado, para cálculo da resistência relativa das partículas ao escape do rúmen, durante a E1 e E2, estado de equilíbrio 24 horas após o início da alimentação. Assim, a resistência relativa apresentada na Figura 2 refere-se apenas à digesta amostrada no tempo de 24 horas.

Quando as partículas tentam escapar do rúmen, existe resistência que depende do tamanho das mesmas (Figura 2). Queiroz et al. (1996) encontraram aumento da resistência relativa das partículas ao escape do rúmen, com efeito quadrático, à medida que se aumentava o tamanho das partículas da digesta. Se 


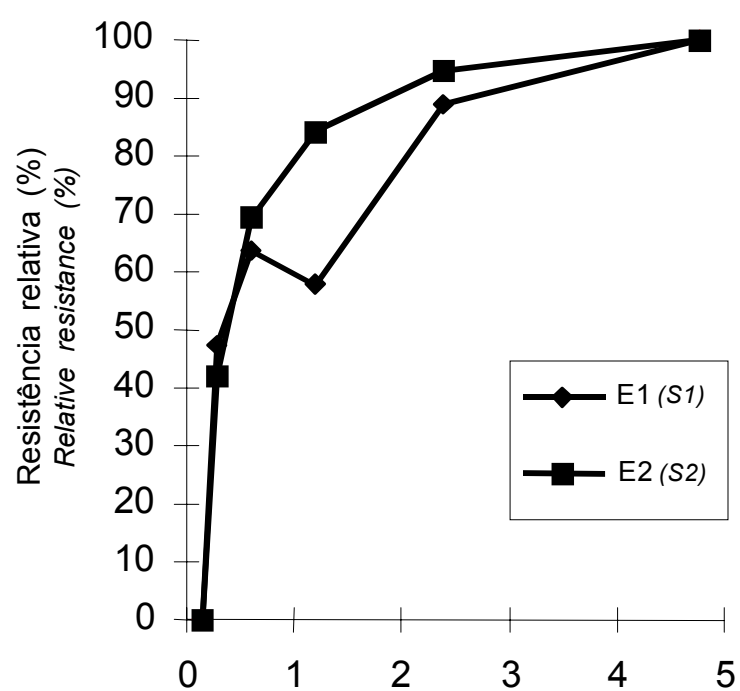

Diâmetro do poro da peneira $(\mathrm{mm})$ Sieve screen $(\mathrm{mm})$

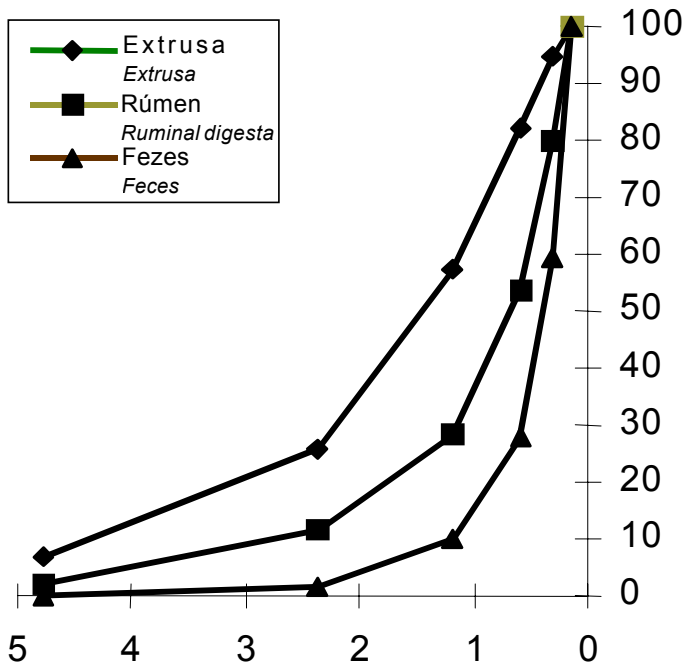

Diâmetro do poro da peneira $(\mathrm{mm})$ Sieve screen $(\mathrm{mm})$

Figura 2 -Resistência relativa das partículas ao escape do rúmen e peso seco acumulado das partículas da extrusa, da digesta ruminal e das fezes de novilhos.

Figure 2 - Relative resistance of the particles to escape from the rumen and acumulative dry weight of particles of the extrusa, ruminal digesta and feces of the steers.

todas as partículas abaixo de certo tamanho crítico escapassem do rúmen com a mesma facilidade, então, a proporção desses diferentes tamanhos de partícula seria o mesmo em ambos conteúdos do rúmen e fezes. Entretanto, há contínuo crescimento na resistência ao escape das partículas do rúmen com o aumento do tamanho da partícula.

Nos gráficos apresentados na Figura 2, nota-se que não há divisão abrupta entre as partículas que podem e, ou, não escapar do rúmen, mas parece existir um ponto de inflexão entre 2,38 e 1,19 mm. Destas, menos de $9 \%$ escapando do rúmen são retidas na peneira de $1,19 \mathrm{~mm}$ e encontram maior resistência ao escape do rúmen na E2 (85\%) que na E1 (58\%). Martz \& Belyea (1986) concluíram que partículas com tamanho menor que 1,2 mm escapam do rúmen, não estando claro se este tamanho varia comforme a espécie de forrageira ou o tipo de alimentação.

O conceito do modelo de dois-pool de partículas implica na homogeneidade dentro dos pools. Entretanto, estes resultados e os apresentados por Poppi et al. (1980) indicam que a facilidade ou probabilidade do escape das partículas dentro destes pools varia com o tamanho da partícula. Por essa razão, quando se utilizar o modelo de dois-pool, deve-se reconhecer que as partículas acima de um tamanho crítico podem escapar do rúmen, embora com grande dificuldade, e que a resistência ao escape varia dentro do espectro do tamanho de partícula.

Além da resistência relativa das partículas ao escape do rúmen, em estado de equilíbrio, a resistência relativa média das partículas ao escape do rúmen, em tempos crescentes após o início da alimentação, foi avaliada. Não houve efeito da época na resistência relativa média, mas foi diferente $(\mathrm{P}<0,01)$ nos tempos de amostragem, com máximo de resistência relativa de $68,34 \%$, às $10,61 \mathrm{~h}$, como pode ser observado pela equação quadrática apresentada na Figura 3. Este valor foi superior aos encontrados por Queiróz et al. (1996), provavelmente porque esses autores trabalharam com bovinos alimentados com dieta contendo $50 \%$ de concentrado e amostragem da digesta realizada após jejum de 16 horas.

Martz \& Belyea (1986) questionaram se a interferência do conteúdo de parede celular na passagem de partícula ocorreu em virtude de as forragens de alta parede celular necessitarem de mais mastigação durante a ruminação ou apresentarem digestão microbiana mais lenta em relação a forragens de baixa parede celular. Caso se acredite na possibilidade de a digestão microbiana das partículas possuir pouca influência na redução do seu tamanho (Van Soest, 
Tabela 3 - Tamanho médio da partícula (TMP) e módulo de finura (MF) das fezes de novilhos mantidos em pastagem natural em diferentes estações do ano

Table 3 - Mean particle size(MPS) and module of fineness (MF) of the feces in steers grazing native pasture in different seasons of the year

\begin{tabular}{|c|c|c|c|c|c|}
\hline & $\begin{array}{l}\text { Época } \\
\text { Season }\end{array}$ & & & $\begin{array}{l}\text { Época } \\
\text { Season }\end{array}$ & \\
\hline & TMP & MF & & TMP & MF \\
\hline & $M P S$ & $F M$ & & $M P S$ & $F M$ \\
\hline Fevereiro & $0,60^{\mathrm{a}}$ & $2,18^{a}$ & Agosto & $0,45^{\mathrm{a}}$ & $1,89^{\mathrm{a}}$ \\
\hline February & & & August & & \\
\hline Março & $0,47^{\mathrm{b}}$ & $1,94^{b}$ & Setembro & $0,46^{\mathrm{a}}$ & $1,91^{\mathrm{a}}$ \\
\hline March & & & September & & \\
\hline Abril & $0,57^{\mathrm{a}}$ & $2,16^{\mathrm{a}}$ & Outubro & $0,45^{\mathrm{a}}$ & $1,88^{\mathrm{a}}$ \\
\hline April & & & October & & \\
\hline Média & $0,55^{\mathrm{A}}$ & $2,09^{\mathrm{A}}$ & Média & $0,45^{\mathrm{B}}$ & $1,90^{\mathrm{B}}$ \\
\hline Mean & & & Mean & & \\
\hline
\end{tabular}

Médias seguidas por letra minúscula diferente, na coluna, diferem $(\mathrm{P}<0,05)$ pelo teste SNK (Means followed by different small letter in the column differ $[P<.05]$ by SNK test).

Médias seguidas por letra maiúscula diferente, na linha, diferem $(P<0,01)$ pelo teste $F$ (Means followed by different capital letters in the row differ $[P<.01]$ by $S N K$ test).

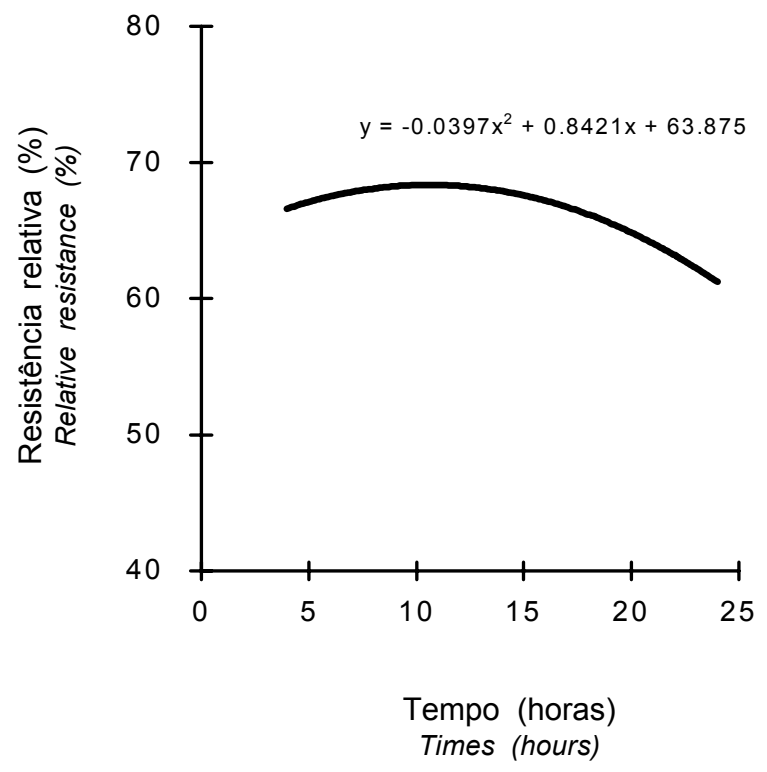

Figura 3 - Estimativa da resistência relativa média (RRM) das partículas à passagem pelo retículo-rúmen de novilhos, em função do tempo de amostragem.

Figure 3 - Estimate of the relative resistance of the particles to passage the rumen-reticulum of the steers, in function of sampling time.

1982; Murphy \& Nicoletti, 1984; Kennedy, 1985; e Ulyatt et al., 1986), a passagem de partículas de forragens de alta parede celular seria influenciada principalmente pela maior necessidade de mastigação.

\section{Conclusões}

O tamanho das partículas mastigadas ingeridas não sofreu variação entre o verão e inverno, estando o tamanho médio da partícula da extrusa próximo de $2 \mathrm{~mm}$.

A redução do tamanho das partículas no inverno é um fator limitante regulando a saída da digesta do rúmen, enquanto no verão favorece o escape de partículas de tamanho médio.

Existe contínuo aumento na resistência ao escape das partículas do rúmen com o aumento do tamanho da partícula.

Partículas maiores que 1,19 mm podem escapar do rúmen, apesar de enfrentarem alta resistência, e partículas menores que este tamanho enfrentam diferentes graus de resistência ao escape.

Diante dos resultados do presente estudo, pode-se inferir, para as condições de pastejo em áreas tropicais, que o manejo das pastagens com intuito de reduzir a maturação das forragens, utilizando-as com menor teor da fração indegradável da parede celular, é uma alternativa para melhorar o consumo voluntário de pasto, que, conseqüentemente, reflete na maior performance animal.

\section{Literatura Citada}

AMERICAN DAIRY SCIENCE ASSOCIATION - ADSA. A report committee on classification of particle size in feedstuffs. Journal of Dairy Science, v.53, n.5, 689-690, 1970.

AMERICAN SOCIETY OF AGRICULTURAL ENGINEERS ASAE. Method of determining and expressing fineness of feed materials by sieving. (Agric.Eng.Yearb., ASAE R246.1) St. Joseph, 1969a. p.346-347.

AMERICAN SOCIETY OF AGRICULTURAL ENGINEERS ASAE. Method of determining modulus of uniformity and modulus of fineness of ground feed. (Agric. Eng. Yearb., ASAE R246.1) St. Joseph, 1969b. p.348.

AMERICAN SOCIETY OF ANIMAL SCIENCE - ASAS. Summary of the minutes of the $61 \mathrm{st}$. Business meeting of the American Society of Animal Science, Journal of Animal Science, v.29, n.2, p.1021-1023, 1969.

BALCH, C.C.; CAMPLING, R.C. Regulation of voluntary food intake in ruminants. Nutrition Abstract and Reviews, v.32, n.3, p.669-686, 1962.

BALDWIN, R.L.; KOONG, L.J.; ULYATT, M.J. A dinamic model of ruminant digestion for evaluation of factors affecting nutritive value. Agricultural Systems, v.2, n.3, p.255-258, 1977.

BURNS, J.C.; FISCHER, D.S.; POND, K.R. et al. Diet characteristics, digesta kinetics, and dry matter intake of steers grazing eastern gamagrass. Journal of Animal Science, v.70, n.4, p.1251-1261, 1992.

CHAI, K.; KENNEDY, P.M.; MILLIGAN, L.P. Reduction in particle size during rumination in cattle. Canadian Journal of Animal Science, v.64, S1, p.339-340, 1984.

COSGROVE, G.P. Grazing behaviour and forage intake. In: GOMIDE, J.A. (Ed.) INTERNATIONAL SYMPOSIUM ON 
ANIMAL PRODUCTION UNDER GRAZING, 1997, Viçosa, MG. Anais...Viçosa, MG: Universidade Federal de Viçosa, 1997. p.59-80.

DULPHY, J.P.; REMOND, B.; THERIEZ, M. Ingestive behaviour and relates activities in ruminants. In: RUCKEBUSCH, Y.; THIVEND, P. (Eds.) Digestive physiology and metabolism in ruminant. Lancaster: MTP Press, 1980. p.103-122.

ENSOR, W.L., OLSON, H.H., COLENBRANDER, V.F. A report: committee on classification of particle size in feedstuffs. Journal of Dairy Science, v.53, n.5, p.689-690, 1970.

EVANS, E.W.; PEARCE, G.R.; BURNETT, J. et al. Changes in some physical characteristic of the digesta in the reticulo-rumen of cows fed once daily. British Journal of Nutrition, v.29, n. 1, p.357-376, 1973.

FAICHNEY, G.J. The kinetics of particulate matter in the rumen. In: MILLIGAN, L.P.; GROVUM, W.L; DOBSON, A. (Eds.) Control of digestion and metabolism in ruminants. INTERNACIONAL SYMPOSIUM ON RUMINANT PHYSIOLOGY, 6., 1984, Canada. Proceedings... Englewood Cliffs: Prentice-Hall, 1986. p.173-195.

FISCHER, D.S.; BURNS, J.C.; POND, K.R. Estimation of mean and median particle size of ruminant digesta. Journal of Dairy Science, v.71, n.2, p.518-524, 1988.

GREENHALGH, J.F.D.; REID, G.W. The effects of pelleting various diets on intake and digestibility in sheep and cattle. Animal Production, v.16, n.3, p.223-233, 1973.

HODGSON, J. Influence of sward characteristics on diet selection and herbage intake by the grazing animal. In: HACKER, J.B. (Ed.) Nutritional limits to animal production from pastures. Queensland: CAB, 1982. p.153-166.

HOYOS, P.; LASCANO, C. Valor nutritivo y preferencia por especies forrajeiras nativas en savanas bien drenadas de los llanos orientales de Colombia. Pasturas Tropicales, v.10, n.2, p.2-10, 1988.

KENNEDY, P.M. Effect of rumination of particle size of rumen digesta by cattle. Australian Journal of Agriculture Research, v.36, n.7, p.819-828, 1985.

LEE, J.A.; PEARCE, G.R. The effectiveness of chewing during eating on particle size reduction of roughages by cattle. Australian Journal of Agriculture Research, v.34, n.7, p.609-618, 1984.

LUGINBUHL, J.M.; POND, K.R.; BURNS, J.C. Changes in ruminal and fecal particle weight distribution of steers fed coastal bermudagrass hay at fron levels. Journal of Animal Science, v.68, n.9, p.2869-2873, 1990.

MARTZ, F.A.; BELYEA, R.L. Role of particle size and forage quality in digestion and passage by cattle and sheep. Journal of Dairy Science, v.69, n.7, p.1996-2008, 1986.

McLEOD, M.N.; MINSON, D.J. Large particle breakdown by cattle eating ryegrass and alfafa. Journal of Animal Science, v.66, n.9, p.992-999, 1988a.

McLEOD, M.N.; MINSON, D.J. Breakdown of large particles in forage by simulated digestion and detrition. Journal of Animal Science, v.66, n.9, p.1000-1007, 1988 b.

McLEOD, M.N.; KENNEDY, P.M.; MINSON, D.J. Resistance of leaf and stem fractions of tropical forage to chewing and passage in cattle. British Journal of Nutrition, v.63, n.1, p.105-119, 1990.

MINSON, D.J.; WILSON, J.R. Prediction of intake as an element of forage quality. In: FAHEY Jr., G.C. (Ed.) Forage quality, evaluation, and utilization. Madison: American Society of Agronomy, 1994. p.533-563.

MURPHY, M.R.; NICOLETTI, J.M. Potential reduction of forage and rumen digesta particle size by microbial action. Journal of Dairy Science, v.67, n.14, p.1221-1226, 1984.

PEARCE, G.D. Changes in particle size in the reticulo-rumen of sheep. Australian Journal of Agricultural Research, v.18, n.1, p.119-125, 1967.

PEREIRA, J.M.; NASCIMENTO Jr., D.; SANTANA, J.R. et al. Teor de proteína bruta e digestibilidade "in vitro" da matéria seca da forragem disponível e da dieta selecionada por bovinos em pastagem de Brachiaria humidicula (Rendle) e leguminosas, submetida a diferentes taxas de lotação. Revista Brasileira de Zootecnia, v.21, n.1, p.104-117, 1992.

POPPI, D.P.; NORTON, B.W.; MINSON, D.J. et al. The validity of the critical size theory for particles leaving the rumen. Journal of Agriculture Science, v.94, n.2, p.275-280, 1980.

POPPI, D.P.; MINSON, D.J.; TERNOUTH, J.H. Studies of cattle and sheep eating leaf and stem fractions of grasses. III. The retention time in the rumen of large feed particles. Australian Journal of Agriculture Research, v.32, n.1, p.123-137, 1981.

QUEIROZ, A.C.; SANCHEZ, N.R.; RESENDE, F.D. et al. Tamanho crítico de partícula e resistência relativa à passagem da partícula da digesta pelo retículo-rúmen, em ruminantes de diferentes grupos genéticos. Revista da Sociedade Brasileira de Zootecnia, v.25, n.3, p.553-567, 1996.

REID, C.S.W.; ULYATT, M.J.; MONRO, J.A. The physical breakdown of feed during digestion in the rumen. Proceedings of New Zealand Society of Animal Production, v.37, n.1, p.173-175, 1977.

REID, C.S.W. The progress of solid feed redidues through the rumino-rreticulum: the ins and outs of paticles. In: BAKER, S.K. et al. (Eds.) Ruminant phisiology concepts and consequences. Murdoch: University Western Australian, 1984. p.79-84.

SMITH, L.W.; WALDO, D.R.; MOORE, L.A. et al. Passage of plant cell wall constituents in sheep. Journal of Dairy Science, v.50, n.7, p.990-995, 1967.

SMITH, L.W.; WALDO, D.R. Method for sizing forage cell wall particles. Journal of Dairy Science, v.52, n.8, p.2051-2053, 1969.

SUTHERLAND, T.M. Particle separation in the forestomachs of sheep. In: DOBSON, A.; DOBSON, M.J. (Eds.) Aspects of digestive physiology in ruminants. Ithaca: Ithaca Comstock Publishing Associates, 1988. p.43-73.

UDÉN, P.; VAN SOEST, P.J. The determination of particle size in some herbivores. Animal Feed Science and Technology, v.7, n.1, p.35-44, 1982.

ULYATT, M.J. Plant fiber and regulation of digestion in the ruminant. In: WALLACE, G.E.; BELL, L. (Eds.) Fiber in human and animal nutrition. Wellington: Royal Society of New Zealand, 1983. p.103-107.

ULYATT, M.J.; DELLOW, D.W.; JOHN, A. et al. Contribution of chewing during eating and rumination as the clearance of digesta from the rumino-reticulum. In: MILLIGAN, L.P.; GROVUM, W.L; DOBSON, A. (Eds.) Control of digestion and metabolism in ruminants. INTERNACIONAL SYMPOSIUM ON RUMINANT PHYSIOLOGY, 6., 1984, Canada. Proceedings... Englewood Cliffs: Prentice-Hall, 1986. p.498-515.

Van SOEST, P.J. Nutritional ecology of the ruminant. Corvalles: O \& B Books Incorporated, 1982. 170p.

Van SOEST, P.J. Nutritional ecology of the ruminant. Ithaca: Cornell University Press, 1994. 470p.

Van SOEST, P.J.; SNIFFEN, C.J.; ALLEN, M.S. Rumen dynamics. In: DOBSON, A.; DOBSON, M.J. (Eds.) Aspects of digestive physiology in ruminants. Ithaca: Ithaca Comstock Publishing Associates, 1988. p.21-42.

WAGHORN, G.C.; SHELTON, E.D.; THOMAS, W.J. Particle breakdown and rumen digestion of fresh ryegrass (Lolium perenne) and lucerne (Medicago sativa) fed to cows during a restricted feeding period. British Journal of Nutrition, v.61, n.2, p.409-423, 1989. 